Annuaire suisse de politique de développement

$14 \mid 1995$

Annuaire Suisse - Tiers Monde 1995

\title{
Problèmes de cohérence dans les relations Suisse - Indonésie
}

Andreas Zürcher

\section{(2) OpenEdition}

1 Journals

Édition électronique

URL : http://journals.openedition.org/aspd/1458

DOI : $10.4000 /$ aspd. 1458

ISSN : 1663-9669

Éditeur

Institut de hautes études internationales et du développement

\section{Édition imprimée}

Date de publication : 1 février 1995

Pagination : 191-193

ISSN : 1660-5934

\section{Référence électronique}

Andreas Zürcher, "Problèmes de cohérence dans les relations Suisse - Indonésie », Annuaire suisse

de politique de développement [En ligne], 14 | 1995, mis en ligne le 03 mai 2013, consulté le 08

septembre 2020. URL : http://journals.openedition.org/aspd/1458 ; DOI : https://doi.org/10.4000/ aspd. 1458

(C) The Graduate Institute | Geneva 


\title{
Problèmes de cohérence dans les relations Suisse - Indonésie
}

\author{
Andreas Zürcher, Ciba-Geigy
}

Remarque préliminaire: Dans cet article et au cours des discussions du 14 septembre 1994, je représente exclusivement mes opinions personnelles, autrement dit pas celles de mon employeur, la société Ciba-Geigy. Par ailleurs, ma façon de voir les choses est, bien évidemment, empreinte de mes trente ans d'expérience professionnelle dans une entreprise multinationale. Ma contribution se rapporte donc en premier lieu à des aspects économiques.

\section{Identification des problèmes de cohérence en général}

a) II me paraît tout d'abord important de noter que dans l'opinion générale d'aujourd'hui deux contradictions n'existent plus, alors qu'elles étaient souvent invoquées comme des problèmes dans les discussions idéologiques à l'époque des tensions Est-Ouest:

- Il est aujourd'hui généralement admis que l'économie privée suisse contribue au développement de pays du Tiers Monde, même si elle y réalise des bénéfices: on n'entend pratiquement plus le grief selon lequel il y aurait "exploitation".

- Il est actuellement admis que les inventions, les marques d'origine et le savoir-faire technologique sont des biens dignes d'être protégés et que l'exercice des droits de monopoles afférents ne peut être assimilé à une exploitation.

b) On peut déboucher sur une contradiction entre l'objectif du développement économique souhaitable et celui d'un compromis favorable à l'environnement. Un tel comportement écologique est certes exigé - et parfois de manière légale - par toutes les parties concernées. Dans d'importants domaines, il repose toutefois sur le volontariat.

Etant donné qu'un comportement écologique délibéré revient en règle générale plus cher à court terme qu'un comportement nocif à l'environnement, les entreprises qui essaient de moins polluer sont très souvent défavorisées sur le plan de la compétitivité. Les "écologiquement bons" se trouvent économiquement sanctionnés et les "écologiquement mauvais" sont économiquement récompensés. Ce paradoxe subsistera aussi longtemps qu'une remise en cause générale du sens donné à l'activité économique n'aura pas lieu.

c) On peut déboucher sur des contradictions entre les exigences "morales" d'aide humanitaire de la part de couches de la population très démunies et 
le postulat selon lequel l'aide au développement devrait contribuer de manière substantielle et durable à la croissance économique. Ces destinataires parmi les plus pauvres de l'aide humanitaire n'ont souvent aucune chance de survie économique dans les conditions qui prévalent après le bouclement d'aide du programme à l'étranger. Des problèmes similaires se posent dans les programmes de formation, dont les participants émigrent pour des raisons économiques (rémunération insuffisante) dans une autre activité professionnelle (par ex. les enseignants en Indonésie).

d) Même les dénonciations justifiées des atteintes aux droits de l'homme provoquent des réactions souvent exacerbées de la part des gouvernements incriminés dans les pays en développement. L'exemple des Hollandais en Indonésie montre précisément que des pays moins pauvres préfèrent renoncer à l'aide économique que de se voir accuser d'atteinte aux droits de l'homme, suite à cette collaboration. Qu'est-ce qui est le plus utile à la population concernée? Devrait-on éventuellement écarter sur le terrain politique (ministère des affaires étrangères) les démarches effectuées pour dénoncer les atteintes aux droits de l'homme? Quelle attitude les entreprises du secteur privé doivent-elles adopter face à des exactions flagrantes dans ce domaine? Un retrait de l'économie privée conduirait en fin de compte à une croissance économique plus lente et, partant, à une pénalisation indirecte de la population.

e) L'exigence de "démocratie" au sens occidental du terme comme condition de base à un Sustainable Development, telle qu'elle est définie dans les directives du Conseil fédéral, me paraît assez loin de la réalité. Dans la plupart des pays en développement, les faits sont bien différents; les gouvernements locaux accordent une oreille plus attentive à des personnages comme Lee Kuan Yu qui ne considère pas une participation politique du peuple comme une condition de base pour une bonne gestion gouvernementale.

Chercher à instaurer des conditions démocratiques dans les pays en développement est sûrement un bon objectif. Je me demande néanmoins s'il est possible d'accorder à cet objectif une importance prioritaire dans le cadre du train de mesures visant à réaliser la cohérence.

\section{Problèmes de cohérence dans le cas de l'Indonésie}

a) Le gouvernement indonésien a assez d'argent pour régler ses principaux problèmes. Ce qui lui manque, ce sont des professionnels bien formés ainsi que des connaissances spécialisées dans de nombreux domaines. Notre aide au développement devrait donc s'articuler autour de la formation de vrais professionnels et de la transmission de connaissances spécialisées. En Indonésie, cela peut toutefois se faire sur une base commerciale. L'Etranger devrait offrir et assurer cette aide, mais en principe moyennant une rétribution. Dans cette optique, le transfert des efforts principaux de la DDA à l'OFAEE me paraît juste. 
b) Si la contribution étrangère au développement de l'Indonésie était conçue conformément au paragraphe a), sur une base commerciale, il faudrait que les subventions fédérales disponibles pour l'Indonésie soient utilisées dans le but de promouvoir les relations bilatérales entre la Suisse et l'Indonésie. Etant donné qu'un développement de ces relations commerciales profite également à l'économie suisse (emplois), nous pouvons, dans le sens de la cohérence, remplir simultanément deux objectifs. Je pense en priorité à des crédits réellement attractifs pour les livraisons/prestations effectuées par des entreprises suisses, telles qu'elles sont accordées dans de grandes proportions par d'autres gouvernements (par exemple le Japon).

c) Le Président de l'Indonésie n'est pas seulement un souverain exerçant un pouvoir politique absolu; par le biais de sa famille, il contrôle d'importants secteurs de l'économie. Dans l'est de l'île de Timor, l'armée indonésienne porte de graves atteintes aux droits de l'homme. Nous avons ainsi en Indonésie toute une kyrielle d'objectifs recherchés - selon les directives du Conseil fédéral - qui ne sont pas remplis. La problématique globale mentionnée au paragraphe $1 \mathrm{~d}$ ) et e) devrait donc être discutée dans le cadre de ce colloque. Personnellement, je ne vois pas de meilleure solution que d'attirer sans cesse, mais avec prudence, l'attention sur notre manière de voir. "Avec prudence" signifie dans ce cas qu'en tant que petit pays, nous n'avons pas suffisamment de prise pour utiliser notre aide au développement ou nos relations commerciales comme moyen de pression.

\section{Mesures pour une meilleure réalisation de la cohérence}

Sur cette question, je ne peux pas fournir un catalogue complet de mesures, mais seulement quelques suggestions dans l'optique particulière de l'économie privée.

a) La prochaine visite du Conseiller fédéral Pascal Delamuraz en Indonésie (octobre 1994) offre sûrement de bonnes possibilités d'expliquer notre point de vue au gouvernement indonésien.

b) Avant la fin de l'année 1994, une Chambre de commerce Suisse-Sud-Est asiatique sera créée. Cette Chambre de commerce peut être utilisée comme forum de discussion sur la cohérence à l'intérieur des entreprises d'exportation suisses ainsi qu'avec les organisations partenaires indonésiennes

c) En Suisse, des efforts sont actuellement déployés pour intensifier la formation et la recherche pour une meilleure connaissance de l'Asie moderne (toute deux sont pour l'instant quasiment inexistantes). Les problèmes de cohérence peuvent être intégrés à ces programmes de formation ou de recherche. Les publics cibles sont les étudiants et les collaborateurs du secteur public et privé qui s'occupent de l'Asie. 